\title{
Single Machine Group Scheduling with Position Dependent Processing Times and Ready Times
}

\author{
Xingong Zhang and Qiulian Xie \\ College of Mathematics Science, Chongqing Normal University, Chongqing 400047, China \\ Correspondence should be addressed to Xingong Zhang; zxg7980@163.com
}

Received 26 January 2015; Revised 11 April 2015; Accepted 12 April 2015

Academic Editor: Chin-Chia Wu

Copyright (C) 2015 X. Zhang and Q. Xie. This is an open access article distributed under the Creative Commons Attribution License, which permits unrestricted use, distribution, and reproduction in any medium, provided the original work is properly cited.

\begin{abstract}
We investigate a single machine group scheduling problem with position dependent processing times and ready times. The actual processing time of a job is a function of positive group-dependent job-independent positional factors. The actual setup time of the group is a linear function of the total completion time of the former group. Each job has a release time. The decision should be taken regarding possible sequences of jobs in each group and group sequence to minimize the makespan. We show that jobs in each group are scheduled in nondecreasing order of its release time and the groups are arranged in nondecreasing order of some certain conditions. We also present a polynomial time solution procedure for the special case of the proposed problem.
\end{abstract}

\section{Introduction}

In traditional scheduling, job processing times are assumed to be fixed and known (Pinedo [1]). However, in reality, we often encounter settings in which the job processing times vary with learning effect (Cheng et al. [2] and Lee et al. [3]), deterioration effect (Alidaee and Womer [4], Cheng et al. [5], and Yin et al. [6]), time dependent effect (Gawiejnowicz [7], Zhang et al. [8], and Yin et al. [9]), and positional effect (Rustogi and Strusevich [10,11], Bachman and Janiak [12], and Yin et al. [13]). Rustogi and Strusevich [14] combined all kinds of effect in a paper which considered the two classical objectives: the makespan and the sum of the completion times.

In the second place, many manufactures have implemented the concept of group technology (GT. Burbidge [15]); it is conventional to schedule continuously all jobs from the same group. Group technology that groups similar products into families helps increase the efficiency of operations and decrease the requirement of facilities (Mitrofanov [16], Janiak and Kovalyov [17], and Webster and Baker [18]). In this paper, we do not assume that all maintenance periods are identical and allow each one of them to leave the processing conditions of the machine in a different state. We deal with a more general concept of a group setup time performed by the processing group. Thus, the effects that change the actual group setup time may become additionally dependent on the actual processing times of jobs before the proposed group.

Rustogi and Strusevich [10] presented real-life examples: "These general positional effects can be found in practice as well. Extending the coursework marking example above, after marking a certain number of scripts, the teacher might get tired or bored, her attention becomes less focused and each new script may even take longer to mark than the one before. We are sure our academic colleagues know this feeling, and they also know the remedy: take a break, have a cup of coffee." However, to the best of our knowledge, only few results concerning scheduling models and problems with position dependent processing times and group technology simultaneously are known. But combining the group technology with start time dependent processing times is more common. For the case that setup time of each group is a fixed constant, Wang et al. [19] considered single machine group scheduling in which the actual processing time of a job is a general linear decreasing function of its starting time; for the makespan minimization problem and total completion time minimization problem they showed that some problems can be solved in polynomial time. $\mathrm{Xu}$ et al. [20] considered the single machine scheduling problems with group technology and ready times; the job processing times are described by a function which is proportional to a linear function of time; 
the setup times of groups are assumed to be fixed and known; it showed that minimizing the makespan with ready times can be solved in polynomial time and proposed a heuristic algorithm. J.-B. Wang and J.-J. Wang [21] continued the work of Xu et al. [20]; they considered a more general deterioration model than the group setup times and job processing times; the objective is to minimize the makespan. They showed that the problem can be solved in polynomial time when start time dependent processing times and group technology are considered simultaneously.

Motivated by the ideas of Rustogi and Strusevich [14] and J.-B. Wang and J.-J. Wang [21], we consider the single machine scheduling problem with ready times of the jobs under the group technology assumption and position dependent processing times. Our objective is to find the optimal group sequence and the optimal job sequence to minimize the makespan. The rest of the paper is organized as follows. In the next section we describe the formulation of our problem. In Section 3, we consider the solution method for minimizing makespan. In Section 4, a reduced model will be introduced and proposed a polynomial time algorithm. The conclusion is given in the last section.

\section{Problem Description}

The independent jobs $J_{1}, J_{2}, \ldots, J_{n}$ have to be processed on a single machine. They are nonpreemptive and to be grouped into $k$ groups: $G_{[1]}, G_{[2]}, \ldots, G_{[k]}$. The jobs in the same group are consecutively processed as long as the job has arrived; a setup time is required if the machine switches from one group to another and all setup times are positive. Assume that each group contains a total of $n_{i}$ jobs, so that the permutation of jobs in the $i$ th group is given by $\pi^{[i]}=$ $\left\{\pi^{[i]}(1), \pi^{[i]}(2), \ldots, \pi^{[i]}\left(n_{[i]}\right)\right\}$, where $\sum_{i=1}^{k} n_{[i]}=n$. Let $r_{\pi^{[i]}(r)}$ represent the ready (arrival) time of the $r$ th job in group $G_{i}$. Depending on the choice of groups and the order in which they are performed, the actual processing time of a job $J_{j}\left(j=\pi^{[i]}(r)\right)$, scheduled in position $r\left(1 \leq r \leq n_{i}\right)$ in group $G_{i}(1 \leq i \leq k)$, is given as follows:

$$
p_{j}^{[i]}(r)=p_{j} g^{[i]}(r), \quad 1 \leq i \leq k, 1 \leq r \leq n_{i},
$$

where $g^{[i]}(r)$ denotes positive group-dependent jobindependent positional factors of the $r$ th job in group $G_{i}$; we call the values $g^{[i]}(r)$ deterioration factors.

Furthermore, since the number of jobs, $n_{i}$, in each group and the total duration of each group, $F_{i}$, are known, the actual setup time $s_{i}$ is defined as follows:

$$
s_{i}=a_{i} F_{i-1}, \quad 1 \leq i \leq k,
$$

where $a_{i}(>0)$ is the deterioration rate of the group $G_{i}$. The deterioration factors $g^{[i]}(r)$ are given in the form of a collection of ordered array of numbers:

$$
1 \leq g^{[i]}(1) \leq g^{[i]}(2) \leq \cdots \leq g^{[i]}\left(n_{i}\right), \quad i=1,2, \ldots, k,
$$

and $r_{\pi^{[i]}(r)}, p_{\pi^{[i]}(r)}$ satisfy the following relations: when $r_{\pi^{[i]}(1)} \leq$ $r_{\pi^{[i]}(2)} \leq \cdots \leq r_{\pi^{[i]}\left(n_{i}\right)}$, there are

$$
p_{\pi^{[i]}(1)} \geq p_{\pi^{[i]}(2)} \geq \cdots \geq p_{\pi^{[i]}\left(n_{i}\right)}, \quad i=1,2, \ldots, k .
$$

For a given schedule $\pi$, let $C_{\pi^{[i]}(r)}=C_{\pi^{[i]}(r)}(\pi)$ denote the completion time of job $J_{\pi^{[i]}(r)}$, and $C_{\max }=\max \left\{C_{\pi^{[i]}(r)} \mid i=\right.$ $\left.1,2, \ldots, k ; r=1,2, \ldots, n_{i}\right\}$ represent the makespan of a given schedule. Using the three-field notation schema in scheduling problems (Graham et al. [22]), the makespan minimization problem is denoted as $1 \mid r_{j}, p_{j}^{[i]}(r)=p_{j} g^{[i]}(r), s_{i}=a_{i} F_{i-1}$, GT $\mid C_{\max }$, where GT denote group technology.

\section{The Solution Method}

In the following section, we will give the solution method so that the single machine minimization scheduling problem with deteriorating jobs and ready times can be solved under certain conditions. Firstly, we consider that all jobs can be processed in one group; that is, $k=1$.

Lemma 1. For the problem $1\left|r_{j}, p_{j}(r)=p_{j} g(r)\right| C_{\max }$, where $g(r)$ meets (3), the optimal job sequence can be obtained by sequencing the jobs in nondecreasing order of $r_{j}$.

Proof. Suppose that $\pi=\left\{S_{1}, J_{i}, J_{j}, S_{2}\right\}$ and $\pi^{\prime}=\left\{S_{1}, J_{j}, J_{i}, S_{2}\right\}$ are two job sequence, where $S_{1}$ and $S_{2}$ denote a partial sequence (note that $S_{1}$ and $S_{2}$ may be empty), and the difference between $\pi$ and $\pi^{\prime}$ is a pairwise interchange of two adjacent jobs $J_{i}$ and $J_{j}$. In addition, the completion time of the last job of $S_{1}$ in sequence $\pi\left(\pi^{\prime}\right)$ is denoted by $A$. Then, the completion times of job $J_{i}$ and $J_{j}$ under $\pi$ are

$$
\begin{aligned}
& C_{i}(\pi)=\max \left\{A, r_{i}\right\}+p_{i} g(r)=\max \left\{A+p_{i} g(r), r_{i}\right. \\
& \left.\quad+p_{i} g(r)\right\}, \\
& C_{j}(\pi)=\max \left\{C_{i}(\pi), r_{j}\right\}+p_{j} g(r+1)=\max \{A \\
& \quad+p_{i} g(r)+p_{j} g(r+1), r_{i}+p_{i} g(r) \\
& \left.\quad+p_{j} g(r+1), r_{j}+p_{j} g(r+1)\right\} .
\end{aligned}
$$

Similarly, the completion times of jobs $J_{j}$ and $J_{i}$ under $\pi^{\prime}$ are

$$
\begin{aligned}
& C_{j}\left(\pi^{\prime}\right)=\max \left\{A, r_{j}\right\}+p_{j} g(r)=\max \{A \\
& \left.\quad+p_{j} g(r), r_{j}+p_{j} g(r)\right\}, \\
& C_{i}\left(\pi^{\prime}\right)=\max \left\{C_{j}\left(\pi^{\prime}\right), r_{i}\right\}+p_{i} g(r+1)=\max \{A \\
& \quad+p_{j} g(r)+p_{i} g(r+1), r_{j}+p_{j} g(r) \\
& \left.\quad+p_{i} g(r+1), r_{i}+p_{i} g(r+1)\right\} .
\end{aligned}
$$

Suppose that $r_{i} \leq r_{j}$, based on (6) and (8), we have

$$
\begin{aligned}
& C_{i}\left(\pi^{\prime}\right)-C_{j}(\pi)=\max \left\{A+p_{j} g(r)+p_{i} g(r+1), r_{j}\right. \\
& \left.\quad+p_{j} g(r)+p_{i} g(r+1), r_{i}+p_{i} g(r+1)\right\}-\max \{A \\
& \quad+p_{i} g(r)+p_{j} g(r+1), r_{i}+p_{i} g(r) \\
& \left.\quad+p_{j} g(r+1), r_{j}+p_{j} g(r+1)\right\} .
\end{aligned}
$$


Based on the formula (3) $p_{i} \geq p_{j}, g(r) \leq g(r+1)$, and the values of $r_{i}, r_{j}$, and $A$, we divide these values into three cases as follows.

(1) $r_{i} \leq r_{j} \leq A$; that is, jobs $J_{i}$ and $J_{j}$ have arrived at time $A$; then

$$
\begin{aligned}
C_{i}\left(\pi^{\prime}\right)-C_{j}(\pi)= & A+p_{j} g(r)+p_{i} g(r+1) \\
& -\left[A+p_{i} g(r)+p_{j} g(r+1)\right] \\
= & \left(p_{i}-p_{j}\right)[g(r+1)-g(r)] \geq 0 .
\end{aligned}
$$

(2) $r_{i} \leq A<r_{j}$; that is, job $J_{i}$ has arrived before time $A$, and $J_{j}$ has not arrived at time $A$; then

$$
\begin{aligned}
C_{i}\left(\pi^{\prime}\right)-C_{j}(\pi)= & r_{j}+p_{j} g(r)+p_{i} g(r+1) \\
& -\left[A+p_{i} g(r)+p_{j} g(r+1)\right] \\
= & \left(r_{j}-A\right) \\
& +\left(p_{i}-p_{j}\right)[g(r+1)-g(r)]>0 .
\end{aligned}
$$

(3) $A<r_{i} \leq r_{j}$; that is, jobs $J_{i}$ and $J_{j}$ have not arrived at time $A$; then

$$
\begin{aligned}
C_{i}\left(\pi^{\prime}\right)-C_{j}(\pi)= & r_{j}+p_{j} g(r)+p_{i} g(r+1) \\
& -\left[r_{i}+p_{i} g(r)+p_{j} g(r+1)\right] \\
= & \left(r_{j}-A\right) \\
& +\left(p_{i}-p_{j}\right)[g(r+1)-g(r)] \\
\geq & 0 .
\end{aligned}
$$

In conclusion, we have $C_{i}\left(\pi^{\prime}\right) \geq C_{j}(\pi)$. Repeating this interchange argument, an optimal schedule can be obtained by sequencing the jobs in nondecreasing order of $r_{j}$.

Next, we consider the problem $1 \mid r_{j}, p_{j}^{[i]}(r)=p_{j} g^{[i]}(r)$, GT | $C_{\text {max }}$; assume that $B$ denotes the completion time of the $(i-1)$ th group and $r_{\pi^{[i]}(1)} \leq r_{\pi^{[i]}(2)} \leq \cdots \leq r_{\pi^{[i]}\left(n_{i}\right)}$ is satisfied in group $G_{i}$. Then we have

$$
\begin{gathered}
C_{\pi^{[i]}(1)}=\max \left\{B+a_{i} B, r_{\pi^{[i]}(1)}\right\}+p_{\pi^{[i]}(1)} g^{[i]}(1) \\
=\max \left\{\left(1+a_{i}\right) B+p_{\pi^{[i]}(1)} g^{[i]}(1), r_{\pi^{[i]}(1)}\right. \\
\left.+p_{\pi^{[i]}(1)} g^{[i]}(1)\right\}, \\
C_{\pi^{[i]}(2)}=\max \left\{C_{\pi_{[i]}(1)}, r_{\pi^{[i]}(2)}\right\}+p_{\pi^{[i]}(2)} g^{[i]}(2) \\
=\max \left\{\left(1+a_{i}\right) B+\sum_{r=1}^{2} p_{\pi_{[i]}(r)} g^{[i]}(r), r_{\pi^{[i]}(1)}\right. \\
\left.+\sum_{r=1}^{2} p_{\pi^{[i]}(r)} g^{[i]}(r), r_{\pi^{[i]}(2)}+p_{\pi^{[i]}(2)} g^{[i]}(2)\right\}, \\
C_{\pi^{[i]}(3)}=\max \left\{C_{\pi^{[i]}(2)}, r_{\pi^{[i]}(3)}\right\}+p_{\pi^{[i]}(3)} g^{[i]}(3)
\end{gathered}
$$

$$
\begin{aligned}
& =\max \left\{\left(1+a_{i}\right) B+\sum_{r=1}^{3} p_{\pi_{[i]}(r)} g^{[i]}(r), r_{\pi^{[i]}(1)}\right. \\
& +\sum_{r=1}^{3} p_{\pi^{[i]}(r)} g^{[i]}(r), r_{\pi_{[i]}(2)}+\sum_{r=2}^{3} p_{\pi_{[i]}(r)} g^{[i]}(r), r_{\pi^{[i]}(3)} \\
& \left.+p_{\pi^{[i]}(3)} g^{[i]}(3)\right\},
\end{aligned}
$$$$
C_{\pi^{[i]}\left(n_{i}\right)}=\max \left\{\left(1+a_{i}\right) B+\sum_{r=1}^{n_{i}} p_{\pi^{[i]}(r)} g^{[i]}(r), r_{\pi^{[i]}(1)}\right.
$$$$
+\sum_{r=1}^{n_{i}} p_{\pi^{[i]}(r)} g^{[i]}(r), r_{\pi^{[i]}(2)}
$$$$
\left.+\sum_{r=2}^{n_{i}} p_{\pi^{[i]}(r)} g^{[i]}(r), \ldots, r_{\pi^{[i]}\left(n_{i}\right)}+p_{\pi^{[i]}\left(n_{i}\right)} g^{[i]}\left(n_{i}\right)\right\}
$$$$
=\max \left\{\left(1+a_{i}\right) B+\sum_{r=1}^{n_{i}} p_{\pi^{[i]}(r)} g^{[i]}(r), r_{\pi^{[i]}(B(i))}\right.
$$$$
\left.+\sum_{r=B(i)}^{n_{i}} p_{\pi^{[i]}(r)} g^{[i]}(r)\right\}
$$

where

$$
\begin{aligned}
& r_{\pi^{[i]}(B(i))}+\sum_{r=B(i)}^{n_{i}} p_{\pi^{[i]}(r)} g^{[i]}(r)=\max \left\{r_{\pi^{[i]}(1)}\right. \\
& +\sum_{r=1}^{n_{i}} p_{\pi^{[i]}(r)} g^{[i]}(r), r_{\pi^{[i]}(2)} \\
& \left.+\sum_{r=2}^{n_{i}} p_{\pi^{[i]}(r)} g^{[i]}(r), \ldots, r_{\pi^{[i]}\left(n_{i}\right)}+p_{\pi^{[i]}\left(n_{i}\right)} g^{[i]}\left(n_{i}\right)\right\}, \\
& B(i) \in\left\{1,2, \ldots, n_{i}\right\}, i=1,2, \ldots, k .
\end{aligned}
$$

Theorem 2. For the problem $1 \mid r_{j}, p_{j}^{[i]}(r)=p_{j} g^{[i]}(r)$, $s_{i}=a_{i} F_{i-1}, G T \mid C_{\max }$, where $g^{[i]}(r)$ satisfies formula (3), and $p_{\pi^{[i]}(r)}, r_{\pi^{[i]}(r)}$ satisfy formula (4). An optimal schedule can be obtained by the conditions as follows.

If (1) jobs in each group are scheduled in nondecreasing order of $r_{\pi^{[i]}(j)}$, that is,

$$
r_{\pi^{[i]}(1)} \leq r_{\pi^{[i]}(2)} \leq \cdots \leq r_{\pi^{[i]}\left(n_{i}\right)}, \quad i=1,2, \ldots, k,
$$

and if (2) the groups are arranged in nondecreasing order of

$$
\begin{gathered}
\frac{r_{\pi^{[i]}(B(i))}-\sum_{r=1}^{B(i)-1} p_{\pi^{[i]}(r)} g^{[i]}(r)}{1+a_{i}} \\
\frac{\sum_{r=1}^{n_{i}} p_{\pi^{[i]}(r)} g^{[i]}(r)}{a_{i}}
\end{gathered}
$$


where

$$
\begin{aligned}
& r_{\pi^{[i]}(B(i))}+\sum_{r=B(i)}^{n_{i}} p_{\pi^{[i]}(r)} g^{[i]}(r)=\max \left\{r_{\pi^{[i]}(1)}\right. \\
& +\sum_{r=1}^{n_{i}} p_{\pi^{[i]}(r)} g^{[i]}(r), r_{\pi^{[i]}(2)} \\
& \left.+\sum_{r=2}^{n_{i}} p_{\pi^{[i]}(r)} g^{[i]}(r) \ldots, r_{\pi^{[i]}\left(n_{i}\right)}+p_{\pi^{[i]}\left(n_{i}\right)} g^{[i]}\left(n_{i}\right)\right\},
\end{aligned}
$$

where $B(i) \in\left\{1,2, \ldots, n_{i}\right\}, i=1,2, \ldots, k$.

Proof. In the same group, the result of (1) can be easily obtained by Lemma 1. Next, we consider the case in item (2).
Let $\pi$ and $\pi^{\prime}$ be a pairwise interchange of two adjacent groups $G_{i}$ and $G_{j}$, that is, $\pi=\left[S_{1}, G_{i}, G_{j}, S_{2}\right]$ and $\pi^{\prime}=$ $\left[S_{1}, G_{j}, G_{i}, S_{2}\right]$, where $S_{1}$ and $S_{2}$ are partial sequences. Further, we assume that $B$ denotes the completion time of the last job in $S_{1}$. To show $\pi$ dominates $\pi^{\prime}$, it suffices to show that $C_{\pi^{[j]}\left(n_{j}\right)}(\pi) \leq C_{\pi^{[i]}\left(n_{i}\right)}\left(\pi^{\prime}\right)$. Under $\pi$, using (13), we obtain that the completion time of group $G_{i}$ is

$$
\begin{aligned}
& C_{\pi^{[i]}\left(n_{i}\right)}(\pi)=\max \left\{\left(1+a_{i}\right) B\right. \\
& \left.\quad+\sum_{r=1}^{n_{i}} p_{\pi^{[i]}(r)} g^{[i]}(r), r_{\pi^{[i]}(B(i))}+\sum_{r=B(i)}^{n_{i}} p_{\pi^{[i]}(r)} g^{[i]}(r)\right\},
\end{aligned}
$$

and the completion time of the group $G_{j}$ is

$$
\begin{aligned}
& C_{\pi^{[j]}\left(n_{j}\right)}(\pi)=\max \left\{\left(1+a_{j}\right) C_{\pi^{[j]}\left(n_{i}\right)}(\pi)+\sum_{r=1}^{n_{j}} p_{\pi^{[j]}(r)} g^{[j]}(r), r_{\pi^{[j]}(B(j))}+\sum_{r=B(j)}^{n_{j}} p_{\pi^{[j]}(r)} g^{[j]}(r)\right\}=\max \left\{\left(1+a_{j}\right)\left(1+a_{i}\right) B\right. \\
& +\left(1+a_{j}\right) \sum_{r=1}^{n_{i}} p_{\pi^{[i]}(r)} g^{[i]}(r)+\sum_{r=1}^{n_{j}} p_{\pi^{[j]}(r)} g^{[j]}(r),\left(1+a_{j}\right)\left[r_{\pi^{[i]}(B(i))}+\sum_{r=B(i)}^{n_{i}} p_{\pi^{[i]}(r)} g^{[i]}(r)\right]+\sum_{r=1}^{n_{j}} p_{\pi^{[j]}(r)} g^{[j]}(r), r_{\pi^{[j]}(B(j))} \\
& \left.+\sum_{r=B(j)}^{n_{j}} p_{j(r)} g^{[j]}(r)\right\}=\max \left\{\max \left\{\left(1+a_{j}\right)\left(1+a_{i}\right) B,\left(1+a_{j}\right)\left[r_{\pi^{[j]}(B(i))}-\sum_{r=1}^{B(i)-1} p_{\pi^{[i]}(r)} g^{[i]}(r)\right]\right\}\right. \\
& \left.+\left(1+a_{j}\right) \sum_{r=1}^{n_{i}} p_{\pi^{[i]}(r)} g^{[i]}(r)+\sum_{r=1}^{n_{j}} p_{\pi^{[j]}(r)} g^{[j]}(r), r_{\pi^{[j]}(B(j))}+\sum_{r=B(j)}^{n_{j}} p_{j(r)} g^{[j]}(r)\right\} .
\end{aligned}
$$

Under $\pi^{\prime}$, the completion time of the group $G_{j}$ is

$$
\left.+\sum_{r=1}^{n_{i}} p_{\pi^{[i]}(r)} g^{[i]}(r), r_{\pi^{[i]}(B(i))}+\sum_{r=B(i)}^{n_{i}} p_{\pi^{[i]}(r)} g^{[i]}(r)\right\}
$$

$$
C_{\pi^{[i]}\left(n_{i}\right)}(\pi)=\max \left\{\left(1+a_{i}\right) B\right.
$$

and the completion time of the group $G_{i}$ is

$$
\begin{aligned}
& C_{\pi^{[i]}\left(n_{i}\right)}\left(\pi^{\prime}\right)=\max \left\{\left(1+a_{i}\right) C_{j n_{j}}\left(\pi^{\prime}\right)+\sum_{r=1}^{n_{i}} p_{\pi^{[i]}(r)} g^{[i]}(r), r_{\pi^{[i]}(B(i))}+\sum_{r=B(i)}^{n_{i}} p_{\pi^{[i]}(r)} g^{[i]}(r)\right\}=\max \left\{\left(1+a_{i}\right)\left(1+a_{j}\right) B\right. \\
& +\left(1+a_{i}\right) \sum_{r=1}^{n_{j}} p_{\pi^{[j]}(r)} g^{[j]}(r)+\sum_{r=1}^{n_{i}} p_{\pi^{[i]}(r)} g^{[i]}(r),\left(1+a_{i}\right)\left[r_{\pi^{[j]}(B(j))}+\sum_{r=B(j)}^{n_{j}} p_{\pi^{[j]}(r)} g^{[j]}(r)\right]+\sum_{r=1}^{n_{i}} p_{\pi^{[i]}(r)} g^{[i]}(r), r_{\pi^{[i]}(B(i))} \\
& \left.+\sum_{r=B(i)}^{n_{i}} p_{\pi^{[i]}(r)} g^{[i]}(r)\right\}=\max \left\{\max \left\{\left(1+a_{i}\right)\left(1+a_{j}\right) B,\left(1+a_{i}\right)\left[r_{\pi^{[j]}(B(j))}-\sum_{r=1}^{B(j)-1} p_{\pi^{[j]}(r)} g^{[j]}(r)\right]\right\}\right. \\
& \left.\quad+\left(1+a_{i}\right) \sum_{r=1}^{n_{j}} p_{\pi^{[j]}(r)} g^{[j]}(r)+\sum_{r=1}^{n_{i}} p_{\pi^{[i]}(r)} g^{[i]}(r), r_{\pi^{[i]}(B(i))}+\sum_{r=B(i)}^{n_{i}} p_{\pi^{[i]}(r)} g^{[i]}(r)\right\} .
\end{aligned}
$$


Suppose that

$$
\begin{aligned}
& \frac{r_{\pi^{[i]}(B(i))}-\sum_{r=1}^{B(i)-1} p_{\pi^{[i]}(r)} g^{[i]}(r)}{1+a_{i}} \\
& \leq \frac{r_{j B(j)}-\sum_{r=1}^{B(j)-1} p_{\pi^{[j]}(r)} g^{[j]}(r)}{1+a_{j}} \\
& \frac{\sum_{r=1}^{n_{i}} p_{\pi^{[i]}(r)} g^{[i]}(r)}{a_{i}} \leq \frac{\sum_{r=1}^{n_{j}} p_{\pi^{[j]}(r)} g^{[j]}(r)}{a_{j}}
\end{aligned}
$$

that is

$$
\begin{aligned}
& \left(1+a_{j}\right)\left[r_{\pi^{[i]}(B(i))}-\sum_{r=1}^{B(i)-1} p_{\pi^{[i]}(r)} g^{[i]}(r)\right] \\
& \leq\left(1+a_{i}\right)\left[r_{\pi^{[j]}(B(j))}-\sum_{r=1}^{B(j)-1} p_{\pi^{[j]}(r)} g^{[j]}(r)\right], \\
& a_{j} \sum_{r=1}^{n_{i}} p_{\pi^{[i]}(r)} g^{[i]}(r) \leq a_{i} \sum_{r=1}^{n_{j}} p_{\pi^{[j]}(r)} g^{[j]}(r) .
\end{aligned}
$$

Based on (19) and (21), we have

$$
\begin{aligned}
& C_{i n_{i}}\left(\pi^{\prime}\right)-C_{j n_{j}}(\pi)=\max \left\{\max \left\{\left(1+a_{i}\right)\left(1+a_{j}\right) B,\left(1+a_{i}\right)\left[r_{\pi^{[j]}(B(j))}-\sum_{r=1}^{B(j)-1} p_{\pi^{[j]}(r)} g^{[j]}(r)\right]\right\}\right. \\
& \left.+\left(1+a_{i}\right) \sum_{r=1}^{n_{j}} p_{j(r)} g^{[j]}(r)+\sum_{r=1}^{n_{i}} p_{\pi^{[i]}(r)} g^{[i]}(r), r_{\pi^{[i]}(B(i))}+\sum_{r=B(i)}^{n_{i}} p_{\pi^{[i]}(r)} g^{[i]}(r)\right\} \\
& -\max \left\{\max \left\{\left(1+a_{i}\right)\left(1+a_{j}\right) B,\left(1+a_{j}\right)\left[r_{\pi^{[i]}(B(i))}-\sum_{r=1}^{B(i)-1} p_{\pi^{[i]}(r)} g^{[i]}(r)\right]\right\}+\left(1+a_{j}\right) \sum_{r=1}^{n_{i}} p_{\pi^{[i]}(r)} g^{[i]}(r)\right. \\
& \left.+\sum_{r=1}^{n_{j}} p_{\pi^{[j]}(r)} g^{[j]}(r), r_{\pi^{[j]}(B(j))}+\sum_{r=B(j)}^{n_{j}} p_{\pi^{[j]}(r)} g^{[j]}(r)\right\} \\
& \geq \max \left\{\max \left\{\left(1+a_{i}\right)\left(1+a_{j}\right) B,\left(1+a_{j}\right)\left[r_{\pi^{[i]}(B(i))}-\sum_{r=1}^{B(i)-1} p_{\pi^{[i]}(r)} g^{[i]}(r)\right]\right\}+\left(1+a_{j}\right) \sum_{r=1}^{n_{i}} p_{\pi^{[i]}(r)} g^{[i]}(r)\right. \\
& \left.+\sum_{r=1}^{n_{j}} p_{\pi^{[j]}(r)} g^{[j]}(r), r_{\pi^{[i]}(B(i))}+\sum_{r=B(i)}^{n_{i}} p_{\pi^{[i]}(r)} g^{[i]}(r)\right\} \\
& -\max \left\{\max \left\{\left(1+a_{i}\right)\left(1+a_{j}\right) B,\left(1+a_{j}\right)\left[r_{\pi^{[i]}(B(i))}-\sum_{r=1}^{B(i)-1} p_{\pi^{[i]}(r)} g^{[i]}(r)\right]\right\}+\left(1+a_{j}\right) \sum_{r=1}^{n_{i}} p_{\pi^{[i]}(r)} g^{[i]}(r)\right. \\
& \left.+\sum_{r=1}^{n_{j}} p_{\pi^{[j]}(r)} g^{[j]}(r), r_{\pi^{[j]}(B(j))}+\sum_{r=B(j)}^{n_{j}} p_{\pi^{[j]}(r)} g^{[j]}(r)\right\}=0
\end{aligned}
$$

Therefore $C_{\pi^{[i]}\left(n_{i}\right)}\left(\pi^{\prime}\right) \geq C_{\pi^{[j]}\left(n_{j}\right)}(\pi)$; this completes the proof.

\section{A Reduced Model}

In this section, we explore a single machine model, which can be expressed as a special case of the general problem $1\left|r_{j}, p_{j}^{[i]}(r)=p_{j} g^{[i]}(r), s_{i}=a_{i} F_{i-1}, \mathrm{GT}\right| C_{\max }$; that is, the actual setup time $s_{i}$ of group $G_{i}$ is a constant $t(>0)$. The main purpose of this section is to give a streamlined conclusion and algorithm. Assume that $B$ denotes the completion time of the $(i-1)$ th group and $r_{\pi^{[i]}(1)} \leq r_{\pi^{[i]}(2)} \leq \cdots \leq r_{\pi^{[i]}\left(n_{i}\right)}$ is satisfied in the group $G_{i}$. Then we have

$$
\begin{aligned}
& C_{\pi^{[i]}\left(n_{i}\right)}=\max \left\{B+t+\sum_{r=1}^{n_{i}} p_{\pi^{[i]}(r)} g^{[i]}(r), r_{\pi^{[i]}(1)}\right. \\
& +\sum_{r=1}^{n_{i}} p_{\pi^{[i]}(r)} g^{[i]}(r), r_{\pi^{[i]}(2)} \\
& \left.+\sum_{r=2}^{n_{i}} p_{\pi^{[i]}(r)} g^{[i]}(r), \ldots, r_{\pi^{[i]}\left(n_{i}\right)}+p_{\pi^{[i]}\left(n_{i}\right)} g^{[i]}\left(n_{i}\right)\right\}
\end{aligned}
$$




$$
\begin{aligned}
& =\max \left\{B+t+\sum_{r=1}^{n_{i}} p_{\pi^{[i]}(r)} g^{[i]}(r), r_{\pi^{[i]}(B(i))}\right. \\
& \left.+\sum_{r=B(i)}^{n_{i}} p_{\pi^{[i]}(r)} g^{[i]}(r)\right\}
\end{aligned}
$$

where

$$
\begin{aligned}
& r_{\pi^{[i]}(B(i))}+\sum_{r=B(i)}^{n_{i}} p_{\pi^{[i]}(r)} g^{[i]}(r)=\max \left\{r_{\pi^{[i]}(1)}\right. \\
& +\sum_{r=1}^{n_{i}} p_{\pi^{[i]}(r)} g^{[i]}(r), r_{\pi^{[i]}(2)} \\
& \left.+\sum_{r=2}^{n_{i}} p_{\pi^{[i]}(r)} g^{[i]}(r), \ldots, r_{\pi^{[i]}\left(n_{i}\right)}+p_{\pi^{[i]}\left(n_{i}\right)} g^{[i]}\left(n_{i}\right)\right\},
\end{aligned}
$$

and $B(i) \in\left\{1,2, \ldots, n_{i}\right\}, i=1,2, \ldots, k$. Then $C_{\pi^{[i]}\left(n_{i}\right)}$ can be written as

$$
\begin{aligned}
C_{\pi^{[i]}\left(n_{i}\right)}= & \max \left\{B+t, r_{\pi^{[i]}(B(i))}-\sum_{r=1}^{B(i)-1} p_{\pi^{[i]}(r)} g^{[i]}(r)\right\} \\
& +\sum_{r=1}^{n_{i}} p_{\pi^{[i]}(r)} g^{[i]}(r) .
\end{aligned}
$$

Theorem 3. For the problem $1 \mid r_{j}, p_{j}^{[i]}(r)=p_{j} g^{[i]}(r), s_{i}=t$, GT | $C_{\max }$ the $g^{[i]}(r)$ satisfies formula (3), and $p_{\pi^{[i]}(r)}, r_{\pi^{[i]}(r)}$ satisfy formula (4); the optimal schedule satisfies the following.

(1) Jobs in each group are scheduled in nondecreasing order of $r_{j}$; that is,

$$
r_{\pi^{[i]}(1)} \leq r_{\pi^{[i]}(2)} \leq \cdots \leq r_{\pi^{[i]}\left(n_{i}\right)}, \quad i=1,2, \ldots, k
$$

(2) The groups are arranged in nondecreasing order of $r_{i B(i)}-\sum_{r=1}^{B(i)-1} p_{\pi^{[i]}(r)} g^{[i]}(r)$, where

$$
\begin{aligned}
& r_{\pi^{[i]}(B(i))}+\sum_{r=B(i)}^{n_{i}} p_{\pi^{[i]}(r)} g^{[i]}(r)=\max \left\{r_{\pi^{[i]}(1)}\right. \\
& +\sum_{r=1}^{n_{i}} p_{\pi^{[i]}(r)} g^{[i]}(r), r_{\pi^{[i]}(2)} \\
& \left.+\sum_{r=2}^{n_{i}} p_{\pi^{[i]}(r)} g^{[i]}(r), \ldots, r_{\pi^{[i]}\left(n_{i}\right)}+p_{\pi^{[i]}\left(n_{i}\right)} g^{[i]}\left(n_{i}\right)\right\},
\end{aligned}
$$

where $B(i) \in\left\{1,2, \ldots, n_{i}\right\}, i=1,2, \ldots, k$.
Proof. Similar to Theorem 2, using the two exchange methods, under $\pi$, based on (27), we can obtain the completion time of $G_{i}$

$$
\begin{aligned}
& C_{\pi^{[i]}\left(n_{i}\right)}(\pi) \\
& \quad=\max \left\{B+t, r_{\pi^{[i]}(B(i))}-\sum_{r=1}^{B(i)-1} p_{\pi^{[i]}(r)} g^{[i]}(r)\right\}
\end{aligned}
$$

$$
+\sum_{r=1}^{n_{i}} p_{\pi^{[i]}(r)} g^{[i]}(r),
$$

and the completion time of $G_{j}$

$$
\begin{aligned}
& C_{\pi^{[i]}\left(n_{j}\right)}(\pi)=\max \left\{C_{\pi^{[i]}\left(n_{i}\right)}(\pi)+t, r_{\pi^{[j]}(B(j))}\right. \\
& \left.-\sum_{r=1}^{B(j)-1} p_{\pi^{[j]}(r)} g^{[j]}(r)\right\}+\sum_{r=1}^{n_{j}} p_{\pi^{[j]}(r)} g^{[j]}(r)=\max \{B \\
& +2 t+\sum_{r=1}^{n_{i}} p_{\pi^{[i]}(r)} g^{[i]}(r)+\sum_{r=1}^{n_{j}} p_{\pi^{[j]}(r)} g^{[j]}(r), r_{\pi^{[i]}(B(i))} \\
& +t+\sum_{r=B(i)}^{n_{i}} p_{\pi^{[i]}(r)} g^{[i]}(r) \\
& \left.+\sum_{r=1}^{n_{j}} p_{\pi^{[j]}(r)} g^{[j]}(r), r_{\pi^{[j]}(B(j))}+\sum_{r=B(j)}^{n_{j}} p_{\pi^{[j]}(r)} g^{[j]}(r)\right\} .
\end{aligned}
$$

Under $\pi^{\prime}$, the completion times of $G_{j}$ and $G_{i}$ are

$$
\begin{aligned}
& C_{\pi^{[j]}\left(n_{j}\right)}\left(\pi^{\prime}\right)=\max \left\{B+t, r_{\pi^{[j]}(B(j))}\right. \\
& \left.-\sum_{r=1}^{B(j)-1} p_{\pi^{[j]}(r)} g^{[j]}(r)\right\}+\sum_{r=1}^{n_{j}} p_{\pi^{[j]}(r)} g^{[j]}(r), \\
& C_{\pi^{[i]}\left(n_{i}\right)}\left(\pi^{\prime}\right)=\max \left\{C_{\pi^{[j]}\left(n_{j}\right)}\left(\pi^{\prime}\right)+t, r_{\pi^{[i]}(B(i))}\right. \\
& \left.+\sum_{r=1}^{B(i)-1} p_{\pi^{[i]}(r)} g^{[i]}(r)\right\}+\sum_{r=1}^{n_{i}} p_{\pi^{[i]}(r)} g^{[i]}(r)=\max \{B \\
& +2 t+\sum_{r=1}^{n_{j}} p_{\pi^{[j]}(r)} g^{[j]}(r) \\
& +\sum_{r=1}^{n_{i}} p_{\pi^{[i]}(r)} g^{[i]}(r), r_{\pi^{[j]}(B(j))}+t \\
& \left.+\sum_{r=B(i)} p_{\pi^{[i]}(r)} g^{[i]}(r)\right\} . \\
& +\sum_{r=B(j)}^{n_{j}} p_{\pi^{[j]}(r)} g^{[j]}(r)+\sum_{r=1}^{n_{i}} p_{\pi^{[i]}(r)} g^{[i]}(r), r_{\pi^{[i]}(B(i))}
\end{aligned}
$$


Supposing that $r_{\pi^{[i]}(B(i))}-\sum_{r=1}^{B(i)-1} p_{\pi^{[i]}(r)} g^{[i]}(r) \leq r_{\pi^{[j]}(B(j))}-$ $\sum_{r=1}^{B(j)-1} p_{\pi^{[j]}(r)} g^{[j]}(r)$, based on (31) and (32), we have

$$
\begin{aligned}
& C_{\pi^{[i]}\left(n_{i}\right)}\left(\pi^{\prime}\right)-C_{\pi^{[j]}\left(n_{j}\right)}(\pi)=\max \{B+2 t \\
& +\sum_{r=1}^{n_{j}} p_{\pi^{[j]}(r)} g^{[j]}(r)+\sum_{r=1}^{n_{i}} p_{\pi^{[i]}(r)} g^{[i]}(r), r_{j B(j)}+t \\
& +\sum_{r=B(j)}^{n_{j}} p_{\pi^{[j]}(r)} g^{[j]}(r)+\sum_{r=1}^{n_{i}} p_{\pi^{[i]}(r)} g^{[i]}(r), r_{\pi^{[i]}(B(i))} \\
& \left.+\sum_{r=B(i)}^{n_{i}} p_{\pi^{[i]}(r)} g^{[i]}(r)\right\}-\max \{B+2 t \\
& +\sum_{r=1}^{n_{i}} p_{\pi^{[i]}(r)} g^{[i]}(r)+\sum_{r=1}^{n_{j}} p_{\pi^{[j]}(r)} g^{[j]}(r), r_{\pi^{[i]}(B(i))}+t \\
& +\sum_{r=B(i)}^{n_{i}} p_{i(r)} g^{[i]}(r)+\sum_{r=1}^{n_{j}} p_{\pi^{[j]}(r)} g^{[j]}(r), r_{\pi^{[j]}(B(j))} \\
& \left.+\sum_{r=B(j)}^{n_{j}} p_{\pi^{[j]}(r)} g^{[j]}(r)\right\}=r_{\pi^{[j]}(B(j))}+t \\
& +\sum_{r=B(j)}^{n_{j}} p_{\pi^{[j]}(r)} g^{[j]}(r)+\sum_{r=1}^{n_{i}} p_{\pi^{[i]}(r)} g^{[i]}(r)-\left[r_{\pi^{[i]}(B(i))}\right. \\
& \left.+t+\sum_{r=B(i)}^{n_{i}} p_{\pi^{[i]}(r)} g^{[i]}(r)+\sum_{r=1}^{n_{j}} p_{\pi^{[j]}(r)} g^{[j]}(r)\right] \\
& =\left[r_{\pi^{[j]}(B(j))}-\sum_{r=1}^{B(j)-1} p_{j(r)} g^{[j]}(r)\right]-\left[r_{\pi^{[i]}(B(i))}\right. \\
& \left.-\sum_{r=1}^{B(i)-1} p_{\pi^{[i]}(r)} g^{[i]}(r)\right] \geq 0
\end{aligned}
$$

Therefore $C_{\pi^{[i]}\left(n_{i}\right)}\left(\pi^{\prime}\right) \geq C_{\pi^{[j]}\left(n_{j}\right)}(\pi)$; this completes the proof.

For the problem $1 \mid r_{j}, p_{j}^{[i]}(r)=p_{j} g^{[i]}(r), s_{i}=t$, GT $\mid C_{\max }$, we provide an algorithm based on the result of Theorem 3 as follows.

\section{Algorithm 4.}

Step 1. Jobs in each group are scheduled in nondecreasing order of $r_{i r}$; that is,

$$
r_{\pi^{[i]}(1)} \leq r_{\pi^{[i]}(2)} \leq \cdots \leq r_{\pi^{[i]}\left(n_{i}\right)}, \quad i=1,2, \ldots, k .
$$

Step 2. Let

$$
\begin{aligned}
& r_{\pi^{[i]}(B(i))}+\sum_{r=B(i)}^{n_{i}} p_{\pi^{[i]}(r)} g^{[i]}(r)=\max \left\{r_{\pi^{[i]}(1)}\right. \\
& +\sum_{r=1}^{n_{i}} p_{\pi^{[i]}(r)} g^{[i]}(r), r_{\pi^{[i]}(2)} \\
& \left.+\sum_{r=2}^{n_{i}} p_{\pi^{[i]}(r)} g^{[i]}(r), \ldots, r_{\pi^{[i]}\left(n_{i}\right)}+p_{\pi^{[i]}\left(n_{i}\right)} g^{[i]}\left(n_{i}\right)\right\},
\end{aligned}
$$

where $B(i) \in\left\{1,2, \ldots, n_{i}\right\}, i=1,2, \ldots, k$.

Calculate $B(i)$ and $r_{\pi^{[i]}(B(i))}-\sum_{r=1}^{B(i)-1} p_{\pi^{[i]}(r)} g^{[i]}(r), i=$ $1,2, \ldots, k$.

Step 3. Groups are scheduled in nondecreasing order of $\rho\left(G_{i}\right)=r_{\pi^{[i]}(B(i))}-\sum_{r=1}^{B(i)-1} p_{\pi^{[i]}(r)} g^{[i]}(r)$.

Obviously, the complexity of obtaining the optimal job sequence within a certain group $G_{i}$ is $O\left(n_{i} \log n_{i}\right)$ and that of obtaining the optimal group sequence is $O(k \log k)$. It is easy to show that $\sum_{i=1}^{k} O\left(n_{i} \log n_{i}\right) \leq O(n \log n)$. Hence, the complexity of Algorithm 4 is at most $O(n \log n)$. In addition, we demonstrate Algorithm 4 by the following example.

Example 5. There are eight jobs $(n=8)$ divided into three groups $(k=3)$ to be processed on a single machine.

Let $s_{i}=3$, and $G_{1}=\left\{J_{\pi^{[1]}(1)}, J_{\pi^{[1]}(2)}\right\}, p_{\pi^{[1]}(1)}=10$, $p_{\pi^{[1]}(2)}=8, g^{[1]}(1)=1, g^{[1]}(2)=1.2, r_{\pi^{[1]}(1)}=3, r_{\pi^{[1]}(2)}=$ $7 ; G_{2}=\left\{J_{\pi^{[2]}(1)}, J_{\pi^{[2]}(2)}, J_{\pi^{[2]}(3)}\right\}, p_{\pi^{[2]}(1)}=9, p_{\pi^{[2]}(2)}=10$, $p_{\pi^{[2]}(3)}=7, g^{[2]}(1)=1.1, g^{[2]}(2)=1.3, g^{[2]}(3)=1.5, r_{\pi_{[2]}(1)}=$ $9, r_{\pi_{[2]}(2)}=2, r_{\pi_{[2]}(3)}=12 ; G_{3}=\left\{J_{\pi^{[3]}(1)}, J_{\pi^{[3]}(2)}, J_{\pi^{[3]}(3)}\right\}$, $p_{\pi^{[3]}(1)}=8, p_{\pi^{[3]}(2)}=5, p_{\pi^{[3]}(3)}=10, g^{[3]}(1)=1, g^{[3]}(2)=1.3$, $g^{[3]}(3)=1.3, g^{[3]}(3)=1.4, r_{\pi^{[3]}(1)}=14, r_{\pi^{[3]}(2)}=15$, $r_{\pi^{[3]}(3)}=3$.

Solution. According to Algorithm 4, we solve Example 5 as follows.

Step 1. The optimal job sequence in $G_{1}, G_{2}, G_{3}$ is

$$
\begin{aligned}
& G_{1}: J_{\pi_{[1]}(1)} \longrightarrow J_{\pi^{[1]}(2)} ; \\
& G_{2}: J_{\pi^{[2]}(2)} \longrightarrow J_{\pi^{[2]}(1)} \longrightarrow J_{\pi^{[2]}(3)} ; \\
& G_{3}: J_{\pi^{[3]}(3)} \longrightarrow J_{\pi_{[3]}(1)} \longrightarrow J_{\pi^{[3]}(2)} .
\end{aligned}
$$

Step 2. Calculating $B(i)$ and $\rho\left(G_{i}\right)$, we compute the following values for each group:

$$
\begin{aligned}
G_{1} & : r_{\pi^{[1]}(B(1))}+\sum_{r=B(1)}^{2} p_{\pi^{[1]}(r)} g^{[1]}(r)=\max \left\{r_{\pi^{[1]}(1)}\right. \\
& \left.+\sum_{r=1}^{2} p_{\pi^{[1]}(r)} g^{[1]}(r), r_{\pi^{[1]}(2)}+p_{\pi^{[1]}(2)} g^{[1]}(2)\right\} \\
& =\max \{3+19.6,7+9.6\}=22.6
\end{aligned}
$$


$B(1)=1$, that is, the jobs from the first to continuous processing in group $G_{1}, \rho\left(G_{1}\right)=r_{\pi^{[1]}((1)}=3$ :

$$
\begin{aligned}
G_{2} & : r_{\pi^{[2]}(B(2))}+\sum_{r=B(2)}^{3} p_{\pi^{[2]}(r)} g^{[2]}(r)=\max \left\{r_{\pi^{[2]}(1)}\right. \\
& +\sum_{r=1}^{3} p_{\pi^{[2]}(r)} g^{[2]}(r), r_{\pi^{[2]}(2)} \\
& \left.+\sum_{r=2}^{3} p_{\pi^{[2]}(r)} g^{[2]}(r), r_{\pi^{[2]}(3)}+p_{\pi^{[2]}(3)} g^{[2]}(3)\right\} \\
& =\max \{2+33.2,9+22.2,12+10.5\}=35.2
\end{aligned}
$$

$B(2)=1$, that is, the jobs from the first to continuous processing in group $G_{2}, \rho\left(G_{2}\right)=r_{\pi^{[2]}(1)}=2$ :

$$
\begin{aligned}
G_{3} & : r_{\pi^{[3]}(B(3))}+\sum_{r=B(3)}^{3} p_{\pi^{[3]}(r)} g^{[3]}(r)=\max \left\{r_{\pi^{[3]}(1)}\right. \\
& +\sum_{r=1}^{3} p_{\pi^{[3]}(r)} g^{[3]}(r), r_{\pi^{[3]}(2)} \\
& \left.+\sum_{r=2}^{3} p_{\pi^{[3]}(r)} g^{[3]}(r), r_{\pi^{[3]}(3)}+p_{\pi^{[3]}(3)} g^{[3]}(3)\right\} \\
& =\max \{3+27.4,14+17.4,15+7\}=31.4
\end{aligned}
$$

$B(3)=2$, that is, the jobs from the second to continuous processing in group $G_{3}, \rho\left(G_{2}\right)=r_{\pi^{[3]}(2)}-p_{\pi^{[3]}(1)} g^{[3]}(1)=4$.

Step 3. Since $\rho\left(G_{2}\right)<\rho\left(G_{1}\right)<\rho\left(G_{3}\right)$, hence, the optimal group sequence is $G_{2} \rightarrow G_{1} \rightarrow G_{3}$.

Therefore, the optimal schedule is $\left[J_{\pi^{[2]}(2)} \rightarrow J_{\pi^{[2]}(1)} \rightarrow\right.$ $\left.J_{\pi^{[2]}(3)}\right] \rightarrow\left[J_{\pi^{[1]}(1)} \rightarrow J_{\pi^{[1]}(2)}\right] \rightarrow\left[J_{\pi^{[3]}(3)} \rightarrow J_{\pi^{[3]}(1)} \rightarrow\right.$ $\left.J_{\pi^{[3]}(2)}\right]$, and the optimal value of the makespan is 90.2 .

\section{Conclusion}

In this paper we have considered the scheduling problem with group technology and position dependent processing times, for the case that group setup times are linearly related to the completion time of the former group and the job processing times are the general nondecreasing function of the positional factors. We showed that the makespan minimization problem with ready times can be solved under certain conditions. A reduced model can be considered as special case of our general model. Furhermore, we present an $O(n \log n)$ time algorithm to solve the proposed problem.

\section{Conflict of Interests}

The authors declare that there is no conflict of interests regarding the publication of this paper.

\section{Acknowledgments}

The authors are grateful to the editor and anonymous referees for their helpful comments on earlier version of this paper. This work was supported by National Natural Science Foundation of China (11401065), the China Postdoctoral Science Foundation funded project (2013M540698 and 2014T70854), The Chongqing Municipal Science and Technology Commission of Natural Science Fund Projects (cstc2014jcyjA00003), and the Key Foundation of Chongqing Normal University (2011XLZ05).

\section{References}

[1] M. Pinedo, Scheduling: Theory, Algorithms, and Systems, Springer, Berlin, Germany, 2008.

[2] T. C. E. Cheng, W. H. Kuo, and D. L. Yang, "Scheduling with a position-weighted learning effect based on sum-of-logarithmprocessing-times and job position," Information Sciences, vol. 221, pp. 490-500, 2013.

[3] W.-C. Lee, C.-C. Wu, and P.-H. Hsu, "A single-machine learning effect scheduling problem with release times," Omega, vol. 38, no. 1-2, pp. 3-11, 2010.

[4] B. Alidaee and N. K. Womer, "Scheduling with time dependent processing times: review and extensions," Journal of the Operational Research Society, vol. 50, no. 7, pp. 711-720, 1999.

[5] T. C. Cheng, Q. Ding, and B. M. Lin, "A concise survey of scheduling with time-dependent processing times," European Journal of Operational Research, vol. 152, no. 1, pp. 1-13, 2004.

[6] Y. Q. Yin, T. C. E. Cheng, L. Wan, C. C. Wu, and J. Liu, "Two-agent single-machine scheduling with deteriorating jobs," Computers \& Industrial Engineering, vol. 81, pp. 177-185, 2015.

[7] S. Gawiejnowicz, Time-Dependent Scheduling, Monographs in Theoretical Computer Science. An EATCS Series, Springer, Berlin, Germany, 2008.

[8] X. G. Zhang, G. L. Yan, and G. C. Tang, "Single-machine scheduling problems with timedependent and positiondependent learning effects," Annals of Operations Research, vol. 186, pp. 345-356, 2011.

[9] Y. Yin, M. Liu, J. Hao, and M. Zhou, "Single-machine scheduling with job-position-dependent learning and time-dependent deterioration," IEEE Transactions on Systems, Man, and Cybernetics A: Systems and Humans, vol. 42, no. 1, pp. 192-200, 2012.

[10] K. Rustogi and V. A. Strusevich, "Single machine scheduling with general positional deterioration and rate-modifying maintenance," Omega, vol. 40, no. 6, pp. 791-804, 2012.

[11] K. Rustogi and V. A. Strusevich, "Simple matching vs linear assignment in scheduling models with positional effects: a critical review," European Journal of Operational Research, vol. 222, no. 3, pp. 393-407, 2012.

[12] A. Bachman and A. Janiak, "Scheduling jobs with positiondependent processing times," Journal of the Operational Research Society, vol. 55, no. 3, pp. 257-264, 2004.

[13] Y. Yin, D. Xu, K. Sun, and H. Li, "Some scheduling problems with general position-dependent and time-dependent learning effects," Information Sciences, vol. 179, no. 14, pp. 2416-2425, 2009.

[14] K. Rustogi and V. A. Strusevich, "Combining time and position dependent effects on a single machine subject to rate-modifying activities," Omega, vol. 42, no. 1, pp. 166-178, 2014. 
[15] J. L. Burbidge, Group Technology in the Engineer Industry, Mechanical Engineering Publication, London, UK, 1980.

[16] S. P. Mitrofanov, Scientific Principles of Group Technology, National Lending Library, London, UK, 1966.

[17] A. Janiak and M. Y. Kovalyov, "Single machine group scheduling with ordered criteria," Annals of Operations Research, vol. 57, pp. 191-201, 1995.

[18] S. Webster and K. R. Baker, "Scheduling groups of jobs on a single machine," Operations Research, vol. 43, no. 4, pp. 692703, 1995.

[19] J.-B. Wang, A.-X. Guo, F. Shan, B. Jiang, and L.-Y. Wang, "Single machine group scheduling under decreasing linear deterioration," Journal of Applied Mathematics \& Computing, vol. 24, no. 1-2, pp. 283-293, 2007.

[20] Y.-T. Xu, Y. Zhang, and X. Huang, "Single-machine ready times scheduling with group technology and proportional linear deterioration," Applied Mathematical Modelling, vol. 38, no. 1, pp. 384-391, 2014.

[21] J.-B. Wang and J.-J. Wang, "Single machine group scheduling with time dependent processing times and ready times," Information Sciences, vol. 275, pp. 226-231, 2014.

[22] R. L. Graham, E. L. Lawler, J. K. Lenstra, and A. H. G. R. Kan, "Optimization and approximation in deterministic sequencing and scheduling: a survey," Annals of Discrete Mathematics, vol. 5, pp. 287-326, 1979. 


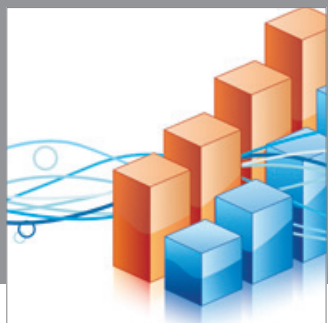

Advances in

Operations Research

mansans

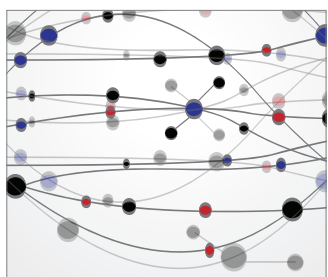

The Scientific World Journal
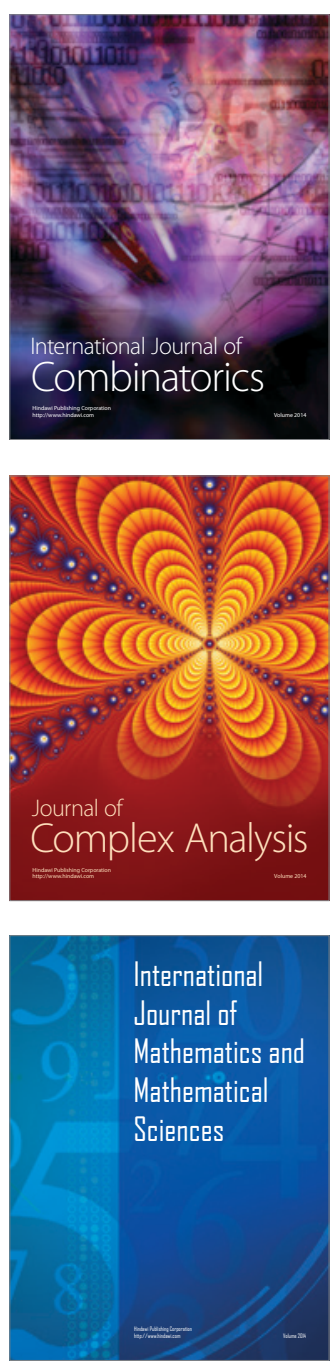
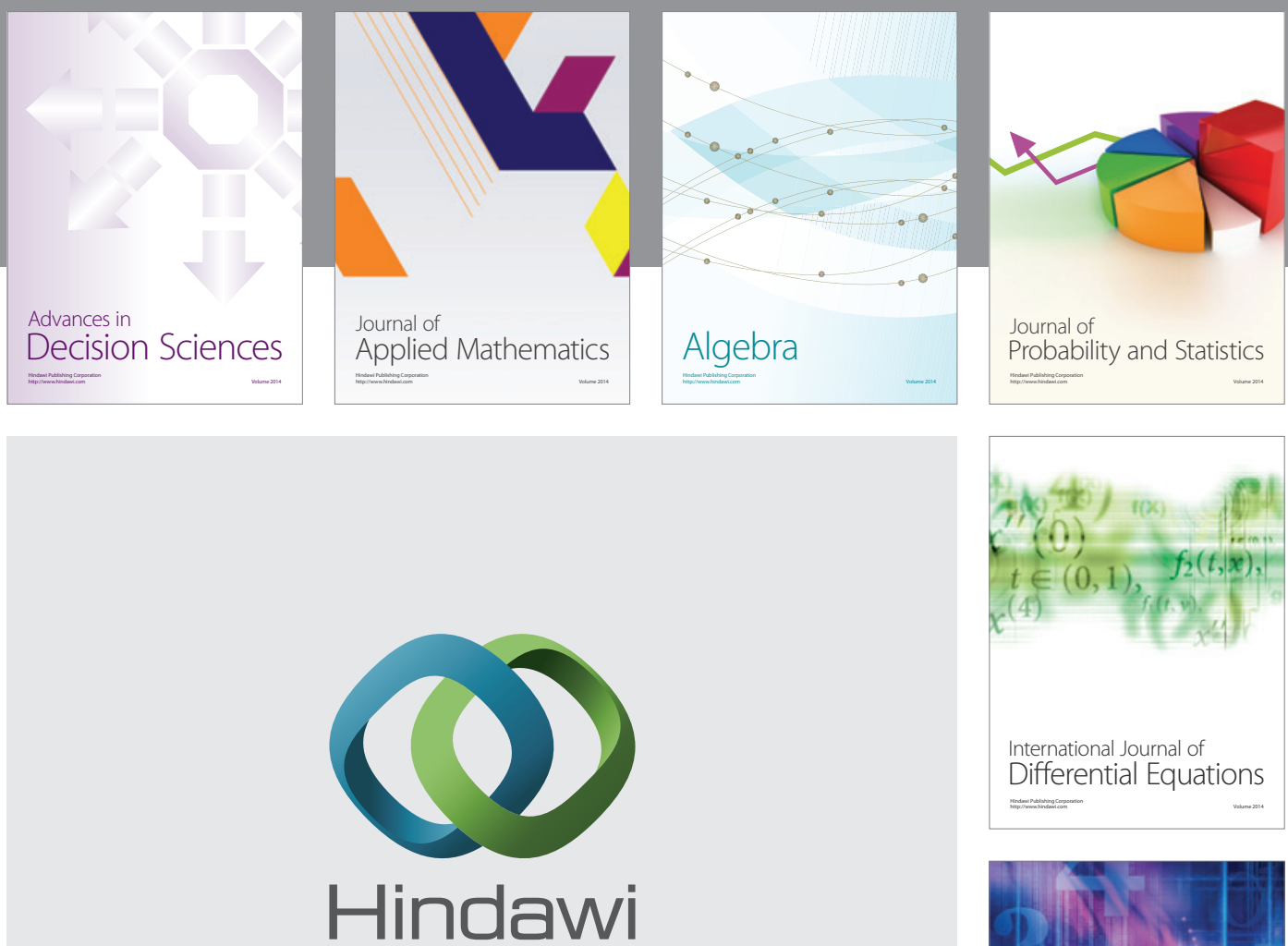

Submit your manuscripts at http://www.hindawi.com
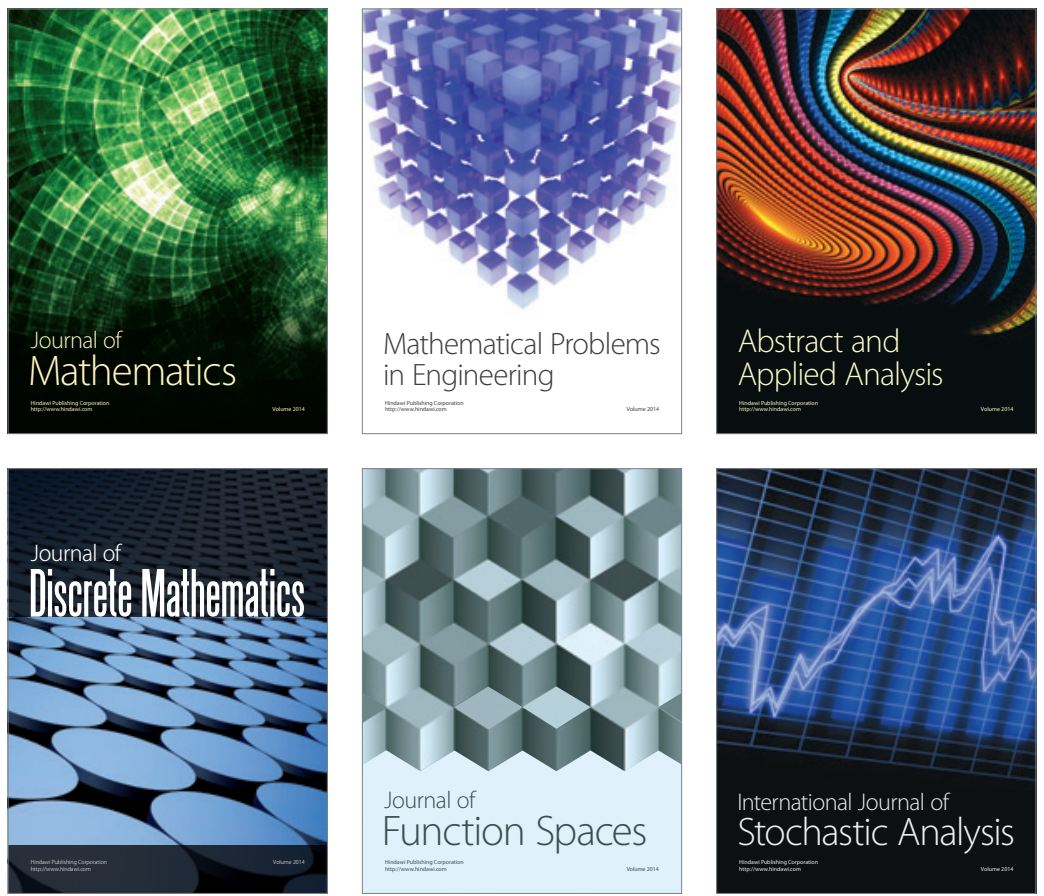

Journal of

Function Spaces

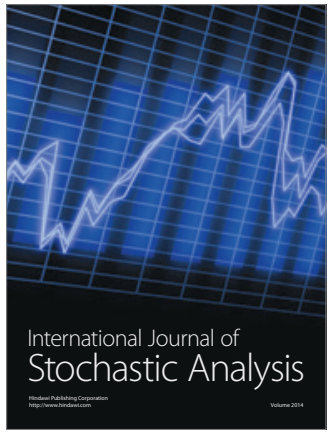

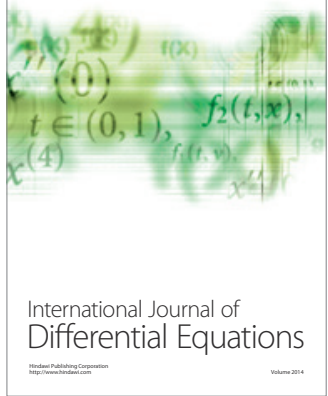
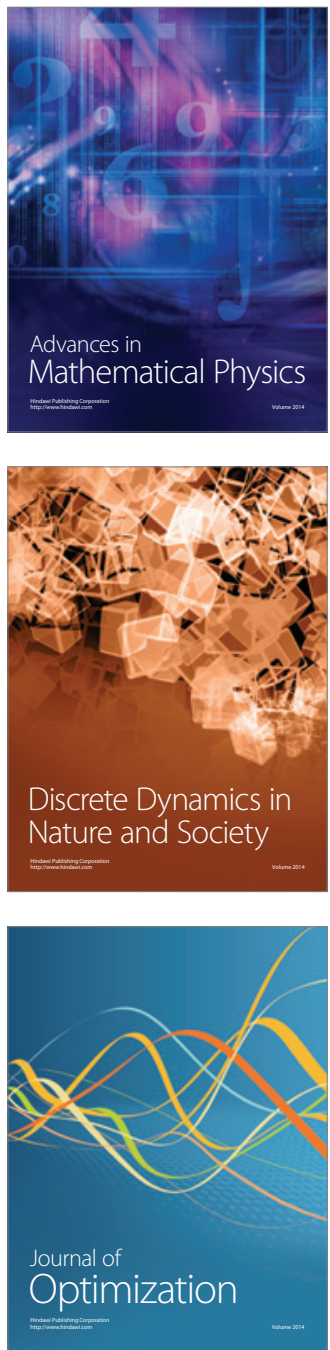